\title{
L'orientation scolaire et professionnelle en Angleterre
}

Advising Pupils about School Options and Careers in England

La orientación escolar y profesional en Inglaterra

Thierry Chevaillier

\section{OpenEdition}

\section{Journals}

Édition électronique

URL : http://journals.openedition.org/ries/1557

DOI : $10.4000 /$ ries. 1557

ISSN : 2261-4265

Éditeur

Centre international d'études pédagogiques

Édition imprimée

Date de publication : 1 avril 2005

Pagination : 95-103

ISSN : 1254-4590

Référence électronique

Thierry Chevaillier, "L'orientation scolaire et professionnelle en Angleterre », Revue internationale

d'éducation de Sèvres [En ligne], 38 | avril 2005, mis en ligne le 01 avril 2008, consulté le 03 mai 2019.

URL : http://journals.openedition.org/ries/1557 ; DOI : 10.4000/ries. 1557

(c) Tous droits réservés 


\section{L'orientation scolaire et professionnelle en Angleterre}

\section{Thierry Chevaillier}

Plutôt que de traiter de l'orientation scolaire dans l'ensemble du Royaume-Uni, nous avons choisi de limiter la présentation et l'analyse à l'Angleterre. En effet, depuis 1999, les quatre pays composant le Royaume-Uni (Angleterre, Écosse, Pays de Galles et Irlande du Nord) ont pris des chemins divergents. Les lois de dévolution ont donné une autonomie substantielle aux trois derniers et ont transféré à leurs assemblées nationales et à leurs exécutifs des compétences étendues, notamment en matière d'éducation et de formation professionnelle. Les choix opérés dans l'organisation des activités d'accueil, d'information et d'orientation (AIO) ont été très différents et il serait trop complexe de présenter les évolutions parallèles des quatre pays.

En outre, l'Angleterre est la composante la plus importante par la taille; elle compte près de 50 millions d'habitants contre 5 millions pour l'Écosse, 3 millions pour le Pays de Galles et 1,5 millions pour l'Irlande du Nord. Enfin, c'est le pays qui a fait les choix les plus radicaux et les plus ambitieux.

Après avoir présenté un rapide historique des transformations récentes et rappelé le cadre législatif de l'orientation scolaire et professionnelle, l'organisation de la profession et le mode de formation des conseillers, nous présenterons plus en détail le système mis en place depuis 2001 avant d'esquisser un premier bilan de son fonctionnement.

\section{RAPIDE HISTORIQUE}

Jusqu'en 1991, les missions d'information et d'orientation (Information, Advice and Guidance) étaient confiées pour l'ensemble du Royaume-Uni aux administrations locales dans le cadre d'un service public national, le Careers Service. Ce service était organisé dans la circonscription de chaque autorité locale d'éducation (Local Education Authority, LEA). Quoiqu'il fût en principe chargé de l'orientation des jeunes scolarisés et des adultes, il concentrait en fait son attention sur les élèves en fin de scolarité obligatoire, aux environs de l'âge de seize ans.

De 1991 à 1995, en Angleterre, en Écosse et au Pays de Galles, le réseau du service public d'orientation a été privatisé : Le ministère britannique de l'éducation et de l'emploi (Department for Education and Employment, comme il était alors nommé) a mis en concurrence des opérateurs locaux, en a sélectionné 
certains et a passé avec eux des contrats à moyen terme. Ainsi, jusqu'en 2000, opéraient soixante-six Careers Service companies. Cependant, malgré le changement de statut des personnels, les usagers privilégiés restèrent les mêmes, ainsi que les méthodes de travail, fondées essentiellement sur les entretiens individuels.

À partir de 1998, l'activité du Careers Service fut recentrée sur les populations «à problèmes » que constituent les jeunes sortis du système éducatif et sans emploi ni formation professionnelle, appelés NEET («not in employment, education or training») ainsi qu'aux élèves en difficulté susceptibles de "décrocher» du système scolaire ou ayant décroché (on notait dans la population une inquiétude croissante au sujet de l'absentéisme à l'école).

C'est cette préoccupation des jeunes en difficulté qui poussa, en 2001, l'Angleterre à transformer complètement son service d'orientation en créant Connexions, un ensemble de partenariats locaux $(C X P)$ mettant en réseau des acteurs publics, associatifs et privés de l'orientation et de l'insertion, avec l'appui d'une forte infrastructure technologique et administrative au plan national, l'Unité nationale de Connexions (CSNU).

\section{LE CADRE LÉGISLATIF}

La loi de 1993 sur la réforme des syndicats et les droits des salariés (Trade Union Reform and Employment Rights Act), a créé pour le ministère de l'Éducation et des Qualifications (Department for Education and Skills, selon la nouvelle appellation), et, à travers lui, pour le Careers Service, l'obligation de fournir des services d'orientation professionnelle à tous les jeunes scolarisés à partir de l'âge de treize ans (à l'exception des étudiants inscrits dans les universités), à tous les jeunes en formation à temps partiel et à tous ceux qui, âgés de moins de vingt-et-un ans, ont quitté le système éducatif depuis moins de deux ans.

Parallèlement, les établissements scolaires qui reçoivent des fonds publics ont un certain nombre d'obligations en matière d'orientation scolaire et professionnelle, fixées par les articles 43 à 45 de la loi sur l'éducation de 1997 : Ils doivent assurer pour les élèves du second cycle du secondaire (de la $9^{\mathrm{e}}$ à la $11^{\mathrm{e}}$ année, soit l'équivalent du lycée pour les Français), des enseignements d'éducation à l'orientation. Cette obligation a été étendue récemment au premier cycle du secondaire. Ces prescriptions sur l'éducation à l'orientation se sont accompagnées dans les années récentes de la définition d'un programme ${ }^{1}$ conseillé mais ne vont cependant pas jusqu'à définir les horaires ou les ressources que l'établissement doit consacrer à l'orientation des élèves. Dans les établissements, des enseignants se sont spécialisés dans l'éducation à l'orientation (careers education teachers).

1. Le ministère finance un site Internet où les enseignants chargés de ces programmes peuvent trouver des informations et des conseils : www.cegnet.co.uk 
Les établissements sont également tenus d'accueillir dans leurs murs les personnels des services d'orientation sous contrat du ministère de l'Éducation afin qu'ils puissent rencontrer les élèves. Ils doivent fournir les locaux et, en collaboration avec le Careers Service, une documentation complète et à jour sur l'orientation et les métiers.

Comme on peut le voir dans ce survol historique, les services d'orientation disposent en Angleterre d'une base législative et réglementaire très claire qui fixe les droits des individus et les obligations des opérateurs et d'une organisation assurant un bon maillage du territoire.

\section{UNE PROFESSION \\ ET UN SECTEUR TRÈS NORMÉS}

Une autre caractéristique du secteur de l'information et de l'orientation en Angleterre est la présence d'organisations professionnelles très structurées à même de fixer et de faire respecter des normes professionnelles et des critères de qualité. Elles définissent les contenus des formations et les niveaux de qualification professionnelle des personnels, en relation avec les établissements d'enseignement supérieur et le ministère de l'éducation et des qualifications.

Ainsi, le Guidance Council ${ }^{2}$, un organisme représentatif de l'ensemble des organisations d'information et d'orientation, a élaboré une charte et des critères de qualité pour les services d'information et d'orientation. Ces critères sont utilisés par la commission d'accréditation de l'orientation (Guidance Accreditation Board) dont l'agrément est obligatoire pour toute organisation recevant des fonds publics pour des activités d'information et d'orientation en Angleterre.

L'institut de l'orientation professionnelle ${ }^{3}$ (Institute of Careers Guidance), l'organisation professionnelle la plus importante exige de ses membres une qualification reconnue soit par un diplôme universitaire, le Diploma in Careers Guidance, soit par un certificat de qualification professionnelle Qualification in Careers Guidance. L'institut a participé à la définition des contenus des formations et des niveaux de qualification professionnelle des personnels, en relation avec les établissements d'enseignement supérieur et le ministère de l'éducation et des qualifications.

Enfin, ces organisations ont produit, avec l'unité nationale de connexions (CSNU) divers instruments d'évaluation interne dont l'usage est recommandé aux organismes faisant partie des partenariats de Connexions pour réaliser les évaluations internes demandées par l'OFSTED.

\footnotetext{
2. www.guidancecouncil.com

3. www.icg-uk.org
} 


\section{LA QUALIFICATION DES PERSON NELS}

La qualification professionnelle ${ }^{4}$ 'sacquiert par la formation initiale et par la voie de l'alternance, de la formation continue ou de la validation des acquis de l'expérience. Son titre le plus élevé est la nouvelle National Vocational Qualification in Advice and Guidance de niveau quatre reconnu au niveau des diplômes universitaires. La formation est assurée par quatorze universités conventionnées avec les organisations professionnelles. Elle se fait sur une base modulaire, chaque module étant défini par les compétences requises dans le métier. Des stages obligatoires s'effectuent durant la formation ou à l'issue de celle-ci.

Une nouvelle voie a été ouverte en 2003, en particulier pour les conseillers personnels employés par Connexions. Elle conduit au National Vocational Qualification in Learning, Development and Support Services. Cette formation se distingue assez nettement de la précédente.

\section{CONNEXIONS, UN NOUVEAU SERVICE}

La décision de restructurer totalement les services d'AIO pour la jeunesse fut argumentée dans deux rapports officiels (white papers) publiés en 1999. Le premier, pour le ministère de l'Éducation était intitulé «apprendre à réussir» (learning to succeed). Il exprimait l'ambition du gouvernement de «donner à tous les jeunes accès à une éducation et à une formation de qualité et de les aider le mieux possible à réussir le passage de l'adolescence à l'âge adulte». Le second rapport, émanant de la cellule sur l'exclusion sociale (SEU), sous le titre de "combler l'écart» (bridging the gap) proposait la création d'un service à compétences multiples à destination de tous les jeunes de treize à dixneuf ans. Ce service devait faire travailler ensemble des administrations publiques, des associations et des entreprises privées spécialisées afin de proposer à chaque jeune un conseiller personnel.

Ce nouveau service, appelé Connexions, fut créé en 2001 mais ne se mit en place progressivement au cours de trois années suivantes. Il se démarque nettement de l'ancienne organisation des services d'orientation et des structures existant dans la plupart des autres pays développés ainsi que de celles mises en place au même moment dans les autres parties du Royaume-Uni ${ }^{5}$.

Réservé aux jeunes de treize à dix-neuf ans, il a en effet été conçu comme un grand «service de la jeunesse » et ne se limite pas à l'orientation mais propose tout un ensemble de services et de conseils (aides financières, logement,

4. Pour un panorama des qualifications et des formations, voir le site de l'ICG www.icg-uk.org/become_an_adviser.html 5. Par exemple, le Pays de Galles a opté pour le maintien d'un service d'orientation et d'insertion pour tous les publics, appelé Careers Wales. 
santé, drogue, contraception, etc.). Pour lutter contre la fragmentation des services destinés à la jeunesse, le programme ambitionne de mettre à la disposition de tous les adolescents un "guichet unique» et des «conseillers personnels» formés au conseil et au travail avec les adolescents qui pourront travailler avec eux et les mettre en relation avec tous les services publics dont ils peuvent avoir besoin, en particulier avec les enseignants et les personnels d'éducation des établissements scolaires, mais aussi avec les services sociaux.

Il est caractérisé ensuite par l'association de structures différentes et un maillage territorial très serré. Il existe quarante-sept partenariats (ou «consortiums») établis au niveau local, sous la responsabilité des Learning and Skills Councils régionaux. Ces partenariats sont constitués par divers services publics du domaine social ou éducatif (appartenant à six départements ministériels), par des entreprises de services ou de conseil et par des associations ou des organisations actives dans ce secteur. Des permanences sont organisées dans des structures de proximité, maisons de quartier, boutiques, aussi bien que dans des établissements scolaires ou des administrations.

Les opérateurs, quel que soit leur statut, reçoivent le droit d'utiliser la «marque» et s'engagent à respecter des principes communs en matière de communication et de formation de leurs personnels. Toute référence au terme Careers, l'appellation traditionnelle des services d'orientation est bannie. Les services aux «clients» sont diversifiés et toutes les méthodes efficaces pour les contacter sont utilisées.

Le site Internet ${ }^{6}$ est un instrument essentiel pour assurer la visibilité de l'organisation mais aussi pour prendre en charge une partie des activités de Connexions: de nombreux services en ligne sont proposés aux jeunes ainsi qu'aux personnels des partenariats locaux.

Connexions fournit aux jeunes qui en font la demande une carte gratuite qui leur propose des services et des réductions sur des achats de services ou de produits sélectionnés et qui leur permet d'accumuler des points en participant à diverses activités. Cette carte, la Connexions card, qui peut également servir à justifier de son âge (dans un pays où la carte d'identité n'existe pas) pourrait prochainement ${ }^{7}$ voir son usage étendu pour favoriser l'accès à des activités sportives et culturelles que le gouvernement voudrait encourager pour lutter contre le désœuvrement et améliorer l'intégration sociale d'une partie de la jeunesse.

Le caractère de service public de Connexions subsiste cependant du fait que l'agrément des partenariats locaux et leur financement reste entre les mains des pouvoirs publics ainsi que le très rigoureux dispositif d'évaluation, confié à

6. Le portail de Connexions connexions.gov.uk ouvre sur trois sites, un réservé aux jeunes, un autre aux professionnels et le troisième à la carte Connexions.

7. Guardian, 15 décembre 2004, "Sport and culture card scheme for teenagers». 
l'OFSTED ${ }^{8}$, l'Office de vérification des standards de l'éducation, par ailleurs chargé de l'évaluation des établissements d'enseignement et des services sociaux pour la jeunesse. Cette évaluation est effectuée régulièrement selon des méthodes clairement présentées aux organismes concernés et au public. Les résultats sont publiés sur le site Internet de l'OFSTED. Dans un souci d'efficacité, le ministère de l'Éducation et des Qualifications est en voie de transférer aux Learning and Skills Councils locaux la responsabilité de l'agrément et du financement des partenariats Connexions.

\section{DU CONSEILLER D'ORIENTATION AU CONSEILLER PERSONNEL}

Un des dispositifs cruciaux du «système » Connexions est le conseiller personnel.

Les fonctions de ce conseiller sont décrites ainsi dans les documents du service :

- aider les jeunes à définir leurs besoins et leur offrir information, conseil et soutien lorsque cela est nécessaire;

- les aider à surmonter les obstacles qui pourraient limiter leur capacité à saisir les occasions d'apprendre ou de travailler;

- collaborer avec les établissements d'enseignement, les organismes de formation et les employeurs pour concevoir et mettre en œuvre des formations adaptées aux besoins de jeunes;

- construire avec les associations, les services publics et les entreprises commerciales une approche d'ensemble pour éliminer les obstacles que rencontrent les jeunes face à l'école ou à l'emploi;

- travailler avec les parents et les familles pour soutenir leurs enfants;

- collecter et traiter efficacement l'information nécessaire à la satisfaction des besoins de jeunes.

Cette description de fonctions s'éloigne de celle des conseillers d'orientation professionnelle telle qu'elle est définie par les organisations professionnelles présentées précédemment. Connexions exige de ses conseillers une formation générale dans le domaine du travail avec les jeunes publics mais pas nécessairement une formation en orientation professionnelle. Certains partenariats recommandent à leur personnel une double formation, mais du fait de la multiplicité des employeurs et des statuts, cet objectif est rarement atteint.

\section{TENSIONS ET DILEMMES ACTUELS}

À partir de 1998, l'accent mis sur l'accueil et le service aux populations «à risque», jeunes chômeurs, élèves en situation d'échec ou en grande difficulté

8. www.ofsted.gov.uk 
conduisit à une nouvelle répartition territoriale des services d'AIO dans les zones économiques déprimées et dans les établissements scolaires aux résultats les plus faibles. Les professionnels de l'orientation scolaire et professionnelle, attachés à un service rendu à tous de manière égale, commencèrent à protester et à déplorer les inégalités de traitement que subissaient les usagers traditionnels du service, les lycéens, pour lesquels un entretien systématique d'orientation au sein de leur établissement ne pouvait plus être garanti.

Dès la création de Connexions, les observateurs avaient souligné la tension qui pouvait naître entre un objectif de couverture générale des besoins de la jeunesse et le choix d'agir de manière sélective en ciblant les actions sur des groupes particuliers.

L'objectif fixé aux partenariats locaux de Connexions de réduire de $10 \%$ le nombre de jeunes de seize à dix-huit ans sans emploi ni formation $(N E E T)$ se traduit souvent sur le terrain par une difficulté pour les établissements scolaires à satisfaire à l'obligation qui leur est faite de fournir à tous les élèves en fin de cycle une aide à l'orientation. Ceci concerne particulièrement les colleges, établissements fréquentés par les jeunes qui poursuivent leurs études au-delà de la scolarité obligatoire et préparent les épreuves du CGSE Advanced Level, examen qui leur permettra l'accès à l'enseignement supérieur.

Le directeur de l'association des collèges, John Brennan, cite, dans un article de presse ${ }^{9}$, une enquête effectuée en 2004 auprès d'institutions dont il a la charge, selon laquelle la moitié des élèves ne bénéficierait pas des services de Connexions et que, pour la plupart de ceux qui y ont accès, la durée est inférieure à une heure par an, ce qui représente une détérioration de la situation par rapport à 2002. Selon lui, c'est la prise en charge des jeunes en difficulté qui absorbe une part trop importante et croissante de l'activité des conseillers.

En 2000, durant la phase de réflexion sur la réforme qui donna naissance à Connexions, les représentants de la profession et les spécialistes avaient exprimé leurs doutes quant à la faisabilité, tant en termes d'organisation que de ressources financières, d'un service universel qui aurait, en particulier, doté chaque jeune de treize à dix-neuf ans d'un conseiller personnel. Leur crainte était que les compétences spécialisées accumulées par le Careers Service ne se diluent et que l'image de l'orientation professionnelle ne se brouille aux yeux du public. Ils ont vu dans l'élargissement des compétences et le changement des qualifications demandées aux nouveaux conseillers une confirmation de leurs craintes.

Mike Tomlinson, président du groupe de travail chargé de préparer la réforme des programmes de l'enseignement secondaire pour les jeunes âgés de quatorze à dix-neuf ans, constatant en février 2004, dans son rapport intéri-

9. The Independent, $1^{\mathrm{er}}$ avril 2004 , «Why is the Connexions service not working ?». 
maire ${ }^{10}$, les lacunes de l'orientation scolaire, souligne que l'extension du bénéfice de services d'information et d'orientation à tous est un facteur-clé de la réussite de la réforme. Recourir pour ce faire aux personnels des établissements scolaires soulève des problèmes importants de moyens et de définition de leur charge de travail.

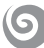

Il est encore trop tôt pour tirer un bilan d'une réforme aussi ambitieuse. Les évaluations faites au niveau local font apparaître des points positifs dans la collaboration d'acteurs de terrain rapprochés dans les partenariats Connexions. Les aspects négatifs fréquemment évoqués concernent la difficulté de faire participer les jeunes à ces nouvelles structures, à leur fonctionnement, leur orientation ou leur évaluation. La fréquentation des nouvelles structures est très inégale selon les régions. Les anciens cloisonnements ne sont pas tous tombés. On souligne la difficulté de faire travailler en réel partenariat les administrations et les organismes du secteur associatif. L'école, qui a semblé un temps négligée comme lieu privilégié de contact avec la jeunesse, pourrait retrouver un rôle plus important dans le cadre d'une nouvelle réforme en préparation dans ce pays où les expériences et les tentatives pour prendre en charge les problèmes de la jeunesse se succèdent à un rythme soutenu.

\section{BIBLIOGRAPHIE}

Department for Education and Employment (1999a): Learning to Succeed. London: The Stationery Office.

Department for Education and Employment (1999b): Schools and Careers Services: Focusing on Individual Needs. London: DfEE.

Social Exclusion Unit (1999). Bridging the Gap: New Opportunities for 16-18 Year Olds Not in Education, Employment or Training. London: Stationery Office.

Centre for Guidance Studies, University of Derby, Research to Inform the Development of the New Connexions Service, 2000 (document contenant les références de nombreuses études récentes menée au Royaume-Uni et en Irlande sur l'exclusion et les problèmes d'orientation).

OCDE, Examen des politiques nationales d'orientation professionnelle, Avril 2003.

10. «14-19 Curriculum and Qualifications Reform », Interim Report of the Working Group on 14-19 Reform, February 2004, www.14-19reform.gov.uk, p.21. 
OECD Review of Career Guidance Policies: United Kingdom, Country Note, 2003.

WATTS A.G., GUICHARD J. PLANT P. et RODRIGUEZ L. (1994): Educational and Vocational Guidance in the European Community. Luxembourg: Office for Official Publications of the European Communities (document plus ancien comparant systèmes d'orientation professionnelle en Europe).

Site Internet de Connexions: connexions.gov.uk

Site Internet de l'OFSTED: www.ofsted.gov.uk 
